\title{
$H$-COBORDANT MANIFOLDS ARE NOT NECESSARILY HOMEOMORPHIC
}

\author{
BY F. T. FARRELL AND W. C. HSIANG ${ }^{1}$
}

Communicated by S. Smale, April 6, 1967

I. Statement of the result. The following two problems are among the outstanding unsolved problems in topology.

1. Is simple homotopy type a topological invariant? (See [4, problem 24], seven classical problems listed by J. W. Milnor.)

2. Let $\left(W ; M, M^{\prime}\right)$ be a (differentiable or piecewise linear) $h$ cobordism. Is $W$ homeomorphic to $M \times[0,1]$ ? (This question also has been asked by many topologists. For example see [9, p. 215].)

These two questions stand at opposite extremes, since an affirmative answer to both leads to an immediate contradiction. In this brief note, we announce that the answer to the second question is negative. Hence, it provides some positive evidence to the first question.

THEOREM. There exists a differentiable (or piecewise linear) $h$-cobor$\operatorname{dism}\left(W^{n+1} ; M^{n}, M^{\prime n}\right)$ for $n \geqq 6$, such that $M^{n}$ is not homeomorphic to $M^{\prime n}$. In particular, $W^{n+1}$ is not homeomorphic to $M^{n} \times I$.

In fact, the manifold $M^{n}$ which is one end of $\partial W^{n+1}$ may be taken as follows. Let $L$ be a 3-dimensional lens space with $\pi_{1}(L)=Z_{p^{2}}$ and let $T^{n-3}$ be the $(n-3)$-dimensional torus. Then, we may take $M$ $=L \times T^{n-3}$.

This theorem is a particular case of a general study concerning (nonsimply connected) $h$-cobordant manifolds which will appear later. We are very grateful to $H$. Bass and M. P. Murthy for sending us their paper [1] and for a very helpful conversation about some algebraic facts.

II. Brief indication of the proof. First, let us recall a theorem of Stallings.

Theorem of Stallings [8]. Let $M^{n}$ be a closed differentiable (or piecewise linear) manifold. For any given element $\tau_{0} \in \mathrm{Wh}\left(\pi_{1} M^{n}\right)$, there exists a differentiable (or piecewise linear) $h$-cobordism $\left(W^{n+1} ; M^{n}, M^{\prime n}\right)$ with $\tau(W, M)=\tau_{0}$.

Second, we shall identify $\pi_{1} M^{n}$ and $\pi_{1} M^{\prime n}$ via their inclusions into

${ }^{1}$ The first named author holds an NSF-graduate fellowship. The second named author is partially supported by NSF-GP-6520. 
$W^{n+1}$ when $\left(W^{n+1} ; M^{n}, M^{\prime n}\right)$ is an $h$-cobordism. Of course, in the general situation when $\pi_{1} M^{n}$ is not abelian, this identification is only defined up to an inner automorphism. But, this will cause no trouble for us, since an inner automorphism induces the identity automorphism on $\mathrm{Wh}\left(\pi_{1} M^{n}\right)$. Besides, when $M^{n}=L \times T^{n-3}, \pi_{1} M^{n}$ is abelian.

Now, we are ready to give a brief indication of the proof. Let us rewrite $M^{n}=L \times T^{n-3}$ as $N^{n-1} \times S^{1}$ where $N^{n-1}=L \times T^{n-4}$. Let $p: M^{n} \rightarrow S^{1}$ be the projection on to the second factor. For any $h$-cobor$\operatorname{dism}\left(W^{n+1} ; M^{n}, M^{\prime n}\right)$, we have a unique map up to homotopy

$$
p^{\prime}: M^{\prime n} \rightarrow S^{1}
$$

which is gotten by the following composite of maps:

$$
M^{\prime n} \stackrel{\text { inclusion }}{\longrightarrow} W^{n+1} \stackrel{\text { retraction }}{\longrightarrow} M^{n} \stackrel{p}{\rightarrow} S^{1} .
$$

Let $X$ be the covering space over $M^{\prime n}$ corresponding to the subgroup $G=\operatorname{ker} p_{\#}^{\prime}$ of $\pi_{1} M^{\prime n} . X$ is an open differentiable (or piecewise linear) manifold with two ends. Hence the obstruction of Novikov-Siebenmann-Wall to splitting $M^{\prime n}$ into $N^{\prime^{n-1}} \times R$ [6] is defined. This obstruction will be denoted by $\sigma\left(p^{\prime}\right)$. On the other hand, we shall denote the obstruction of [2], [3] to altering $p^{\prime}$ (within its homotopy class) to a differentiable (or piecewise linear) fibration by $c\left(p^{\prime}\right)$.

Using the calculation of [1] and the quoted theorem of Stallings, we can find an $h$-cobordism $\left(W^{n+1} ; M^{n}, M^{\prime n}\right)$ such that $\sigma\left(p^{\prime}\right)=0$ but $c\left(p^{\prime}\right) \neq 0$. We claim that $M^{\prime n}$ is not topologically homeomorphic to $M^{n}$. If it were, we could choose a homeomorphism $h: M^{\prime n} \rightarrow M^{n}$ carefully such that the composite map

$$
q: M^{\prime} \stackrel{h}{\rightarrow} M^{n} \stackrel{p}{\rightarrow} S^{1}
$$

is in the same homotopy class as $p^{\prime}$. Let $U$ be a small open interval in $S^{1}$. Then $V=q^{-1}(U)$ is an open submanifold of $M^{\prime n}$ homeomorphic to $N^{n-1} \times R$.

Lift $V$ to $X$. (See Figure 1.)

Using the fact that $\sigma\left(p^{\prime}\right)=0$, one observes that $V$ is also differentiably (or piecewise linearly) splittable, i.e., $V$ is differentiably (or piecewise linearly) expressible as $N^{\prime^{n-1}} \times R$. Put $V=N^{\prime n-1} \times R$ back into $M^{\prime n}$ (See Figure 2.)

Then, $N^{\prime n-1}$ is a differentiable (or piecewise linear) submanifold of $M^{\prime n}$ such that the manifold $M^{\prime}{ }_{N}{ }^{\prime}$ gotten from $M^{\prime n}$ by cutting through $N^{\prime n-1}$ is a differentiable (or piecewise linear) $h$-cobordism. By the very definition of $c\left(p^{\prime}\right)[2],[3]$, we would have $c\left(p^{\prime}\right)=0$. This is a contradiction. 


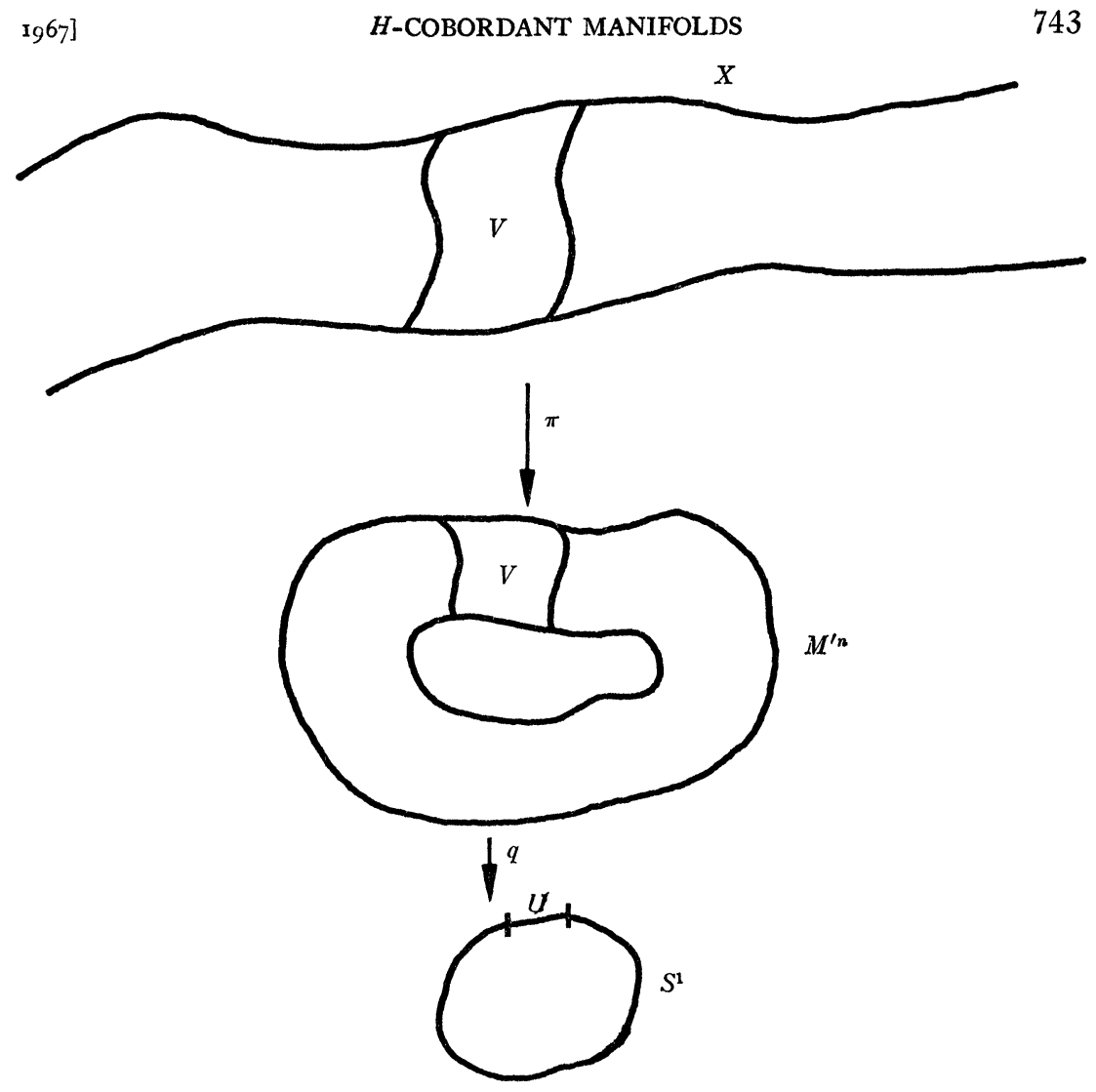

FIGURE 1

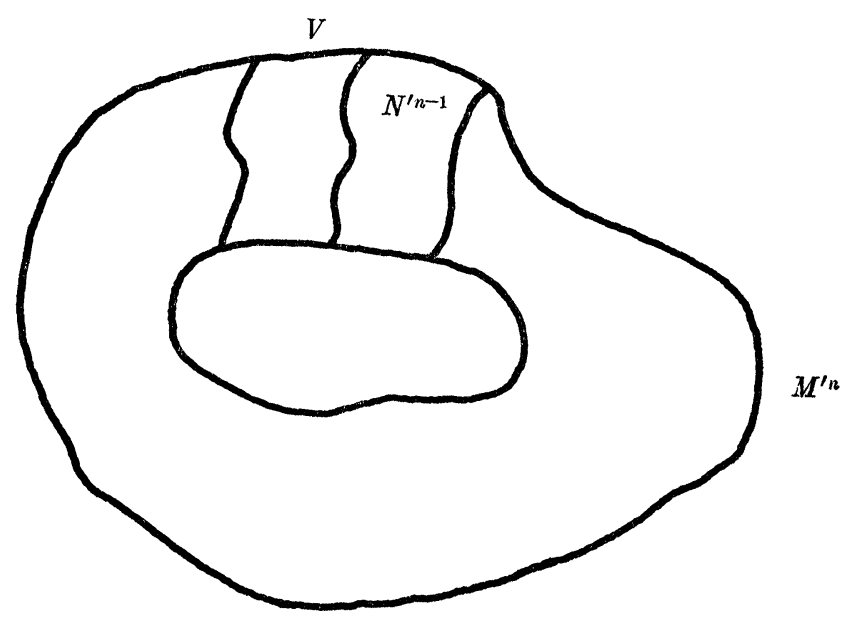

Figure 2 


\section{REFERENCES}

1. H. Bass and M. P. Murthy, Grothendieck groups and Picard groups of abelian group rings, (to appear).

2. F. T. Farrell, The obstruction to fibering a manifold over a circle, Bull. Amer. Math. Soc. 73 (1967), 737-752.

3. - Ph.D. Thesis, Yale University, New Haven, Conn., 1967.

4. R. Lashof (editor), Problems in differential and algebraic topology, Seattle Conference 1963, Ann. of Math. (2)81 (1965), 565-591.

5. J. W. Milnor, Whitehead torsion, Bull. Amer. Math. Soc. 72 (1966), 358-426.

6. S. P. Novikov, Pontryagin classes, the fundamental group and some problems of stable algebras (mimeographed) Internat. Congr. Math., 1966.

7. L. Siebenmann, The obstruction to finding a boundary for an open manifold of dimension greater than five, Ph.D. Thesis, Princeton University, Princeton, N. J., 1965.

8. J. Stallings, On infinite processes leading to differentiability in the complement of a point, Differential and Combinatorial Topology, (A Syposium in honor of M. Morse), Princeton Univ. Press, Princeton, N. J., 1965.

9. R. H. Szczarba, Whithead torsion and h-cobordism, Topology Seminar Wisconsin, 1965, pp. 211-216; Annals of Mathematics Studies, Princeton Univ. Press, Princeton, N. J., 1966.

YALE University 